\title{
Twisted Non-Abelian Determinants on Riemann Supersurfaces
}

\author{
Kenichiro Aoki ${ }^{1 \star}$ and Vipul Periwal ${ }^{2, \star \star}$ \\ ${ }^{1}$ Department of Physics, University of California, Los Angeles, California 90024-1547, USA \\ ${ }^{2}$ Institute for Theoretical Physics, University of California, Santa Barbara, California 93106, USA
}

\begin{abstract}
We calculate the twisted supersymmetric determinants that are necessary for the study of the propagation of spinning strings on arbitrary orbifolds. We calculate the degeneration behaviour of multiloop amplitudes for twisted internal sectors. We use this to derive the spectrum of dimensions of twist fields.
\end{abstract}

In the present work, we shall calculate the superdeterminants of twisted Laplacians acting on tensors of arbitrary weight on hyperbolic supersurfaces. These describe the partition function of spinning strings on backgrounds that arise as quotient spaces of the action of arbitrary finite groups on Euclidean space. Recall that the partition function is given by a measure on moduli space that has the generic form $d \mu_{W P}$ DET, where $d \mu_{W P}$ is the Weil-Petersson measure, and DET stands for a product of superdeterminants (raised to various powers, depending on the string background) of Laplacians on the Riemann surface. The Selberg trace formula applied to appropriate heat kernels, when combined with the Mellin transform relating heat kernels to $\zeta$-functions, allows a simple calculation of these determinants, which we view as defined by derivatives of these $\zeta$-functions at 0 . We shall use the supersymmetric form of the Selberg trace formula obtained in [1]. Unfortunately, these techniques preclude the investigation of left-right asymmetric backgrounds. We believe that some of the techniques employed in the present work should be applicable in more general contexts. Our results are of independent mathematical interest.

Consult the excellent review of D'Hoker and Phong [2] for background, and $[1,3]$ for a discussion of the supersymmetric Selberg formula. Some general references for the Selberg trace formula are $[4,5]$. Twisted determinants were also considered in [6]. We use the conventions of [1] ${ }^{1}$. We shall not bother with explicit

\footnotetext{
* Bitnet: aoki@uclaph. On leave of absence from Department of Physics, Princeton University, Princeton, New Jersey 08544, USA

$\star \star$ Bitnet: viper@sbitp

${ }^{1}$ Changes are indicated explicitly
} 
mention of the prefix "super-" for the most part, except by using a lower-case $s$ where appropriate.

The Riemann surfaces considered are finite-area quotients of the (fixed-pointfree) actions of discrete subgroups, $s \Gamma$, of $\operatorname{OSp}(2 \mid 1, \mathbf{R}) /\{ \pm 1\}$ on the Poincaré superhalf-plane, $s H$. The operators of interest act on spaces of automorphic forms of weight $(n / 2,-n / 2)$ defined as

$$
\mathscr{H}_{n, v} \equiv\left\{f \mid f(\gamma Z)=v_{\gamma}^{2 n}\left(\frac{c z+d+\beta \theta}{c \bar{z}+d+\beta \bar{\theta}}\right)^{n} f(Z), \gamma \in s \Gamma\right\}
$$

here, $v$ is the multiplier system that incorporates the spin structure in this uniformization.

The heat kernel for the square of the Laplacian $\Delta_{n} \equiv 4\left(D_{+} D_{-}^{(n)}\right)^{2}$ acting on $\mathscr{H}_{n, v}$ was constructed in [1]. We shall need the expression for the heat kernel $^{2}$ on $s H$. Define

$$
g_{n}^{t}(r) \equiv \frac{1}{2 \pi^{3 / 2} t^{1 / 2}} \int_{r}^{\infty} \frac{d b \exp \left(-b^{2} / 4 t\right) \sinh (n-1 / 2) b}{\sqrt{2(\cosh b-\cosh r)}} T_{2 n}\left(\frac{\cosh b / 2}{\cosh r / 2}\right),
$$

and let

$$
h_{n}^{t}(r) \equiv-\frac{\sinh r / 2}{4 \pi^{3 / 2} t^{3 / 2}} \int_{r}^{\infty} \frac{b d b \exp \left(-b^{2} / 4 t\right) \cosh n b}{\sqrt{2(\cosh b-\cosh r)}} T_{2 n-1}\left(\frac{\cosh b / 2}{\cosh r / 2}\right)+n \tanh \frac{r}{2} g_{n}^{t}(r)
$$

The heat kernel (after conjugation by $\left.\left(z_{12} / z_{21}\right)^{n}\right)$ on $s H$ is then

$$
G_{n}^{t}\left(Z_{1}, Z_{2}\right)=\left[g_{n}^{t}(r)+i \Delta \bar{\Delta} h_{n}^{t}(r)\right],
$$

where $\cosh r\left(Z_{1}, Z_{2}\right) \equiv 1-2\left(z_{12} z_{\overline{1} \overline{2}} / z_{1 \overline{1}} z_{2 \overline{2}}\right), z_{a b} \equiv z_{a}-z_{b}-\theta_{a} \theta_{b}$,

with

$$
\Delta \equiv \frac{\Theta_{12 \overline{2}}}{\left(z_{12} z_{2 \overline{2}} z_{\overline{2} 1}\right)^{1 / 2}}, \quad \bar{\Delta} \equiv \frac{\Theta_{\overline{1} \overline{2} 2}}{\left(z_{\overline{1} \overline{2}} z_{\overline{2} 2} z_{\overline{2} \overline{1}}\right)^{1 / 2}},
$$

$$
\Theta_{a b c} \equiv \theta_{a} z_{b c}+\theta_{b} z_{c a}+\theta_{c} z_{a b}+\theta_{a} \theta_{b} \theta_{c},
$$

and $T_{k}$ denotes the $k^{\text {th }}$ Chebyshev polynomial.

Let $V$ be a real vector space and $\eta$ be an inner product on $V$. This defines a group of isometries, $S O(V, \eta)$. Let $G$ be a discrete subgroup of $S O(V, \eta)$. Consider a string worldsheet defined by a Fuchsian group, $s \Gamma$. A twisted sector for string propagation with such a worldsheet on $V / G$ is defined by a homomorphism from $s \Gamma$ to $G$, up to automorphisms of the homotopy group of the surface and automorphisms of $G$. Let $\alpha$ denote such a homomorphism. The physical meaning of $\alpha$ is the following: upon going around a curve on the surface that belongs to the homotopy class of some element $g \in s \Gamma$, the image of the worldsheet in spacetime (here $V / G$ ) only closes up to the action of $\alpha(g)$ on $V$.

We are considering the spinning string mapped into $V / G$. The partition function for string propagation on this orbifold is given by a sum over twisted sectors. To obtain the partition function for the twisted sector corresponding to $\alpha$, we wish to

\footnotetext{
${ }^{2}$ All subscripts $n$ in the present on Laplacians, heat kernels $\left(g_{n}, h_{n}, G_{n}, K_{n}\right)$ correspond to $-n$ in [1]
} 
utilize the relationship mentioned at the beginning of this note between determinants and heat kernels. Hence, we wish to construct a heat kernel appropriate for a twisted sector. The physical idea of how to do this is clear: we should twist the image charges according to $\alpha$. We do this by defining

where

$$
\begin{aligned}
K_{n, v, \alpha}^{t}\left(Z, Z^{\prime}\right) \equiv & \sum_{\gamma \in s \Gamma} v_{v}^{2 n}\left(\frac{c z^{\prime}+d+\beta \theta^{\prime}}{c \bar{z}^{\prime}+d+\beta \bar{\theta}^{\prime}}\right)^{n} \\
& \cdot\left(\frac{\gamma z^{\prime}-\bar{z}+\gamma \theta^{\prime} \bar{\theta}}{z-\gamma \bar{z}^{\prime}+\theta \gamma \bar{\theta}^{\prime}}\right)^{n} \alpha(\gamma) \otimes G_{n}^{t}\left(Z, \gamma Z^{\prime}\right),
\end{aligned}
$$

$$
\gamma \equiv\left(\begin{array}{lll}
a & b & \alpha \\
c & d & \beta \\
\delta & \varepsilon & A
\end{array}\right):(z, \theta) \mapsto\left(\frac{a z+b+\alpha \theta}{c z+d+\beta \theta}, \frac{\delta z+\varepsilon+A \theta}{c z+d+\beta \theta}\right) .
$$

Define

$$
\mathscr{H}_{n, v, \alpha} \equiv\left\{f \mid f(\gamma Z)=\alpha(\gamma) v_{\gamma}^{2 n}\left(\frac{c z+d+\beta \theta}{c \bar{z}+d+\beta \bar{\theta}}\right)^{n} f(Z), \gamma \in s \Gamma\right\},
$$

where $f$ are functions on $s H$ that take values in $V . K_{n, v, \alpha}^{t}$ is a map from $\mathscr{H}_{n, v, \alpha} \times \mathscr{H}_{-n, v, \alpha} \rightarrow \mathbf{R}$, the twisted analogue of $K_{n, v}^{t}: \mathscr{H}_{n, v} \times \mathscr{H}_{-n, v} \rightarrow \mathbf{R}$.

Now consider

$$
s \operatorname{Tr} \exp \left(-t \Delta_{n, \alpha}\right) \equiv \int_{s H / s \Gamma} E d^{2} z d^{2} \theta K_{n, v, \alpha}^{t}(Z, Z) \equiv d_{\alpha} I_{n}^{e}(t)+I_{n, \alpha}(t),
$$

where $d_{\alpha}$ is the dimension of $V$, and we have defined

$$
I_{n}^{e}(t) \equiv \int_{s H / s \Gamma} E d^{2} z d^{2} \theta G_{n}^{t}(Z, Z)
$$

and

$$
I_{n, \alpha}(t) \equiv \sum_{\gamma \in S \Gamma \backslash\{\}} v_{\gamma}^{2 n} \operatorname{tr} \alpha(\gamma) \int_{s H / s \Gamma}\left(\frac{c z+d+\beta \theta}{c \bar{z}+d+\beta \bar{\theta}}\right)^{n}\left(\frac{\gamma z-\bar{z}+\gamma \theta \bar{\theta}}{z-\gamma \bar{z}+\theta \gamma \bar{\theta}}\right)^{n} G_{n}^{t}(Z, \gamma Z) .
$$

The calculations in [1] may be modified, using the fact the trace is a class function, to arrive at

$$
\begin{aligned}
I_{n, \alpha}(t)= & \sum_{\gamma \text { primitive }} \sum_{p=1}^{\infty} \frac{l_{\gamma} v_{\gamma}^{2 n p} \operatorname{tr} \alpha\left(\gamma^{p}\right)}{\sqrt{4 \pi t} \sinh \left(p l_{\gamma} / 2\right)} \\
& \cdot \exp \left(-\left(p l_{\gamma}\right)^{2} / 4 t\right)\left[\cosh \left(n-\frac{1}{2}\right) p l_{\gamma}-v_{\gamma}^{p} \cosh n p l_{\gamma}\right],
\end{aligned}
$$

where $l_{\gamma}$ is defined by $2 \cosh l_{\gamma} / 2 \equiv a+d+v_{\gamma} \alpha \beta$, defined by $\gamma \in s \Gamma$. $l_{\gamma}$ is the analogue of the length of the shortest geodesic in the homotopy class defined by $\gamma$ for the supersurface. (An element $\gamma(\neq e)$ in $s \Gamma$ is primitive if it is the positive power of itself alone in $s \Gamma$.) There is, of course, no change in $I_{n}^{e}(t)$ from the untwisted case [1],

$$
I_{n}^{e}(t)=\frac{\chi(s H / s \Gamma)}{2} \sum_{k=0}^{2|n|} \exp \left(k^{2} t\right)
$$

Turning now to the $\zeta$-function, we define $\zeta_{n, \alpha}(s ; c) \equiv d_{\alpha} \zeta_{n}^{e}(s ; c)+\zeta_{n, \alpha}^{r}(s ; c)$, where 


$$
\zeta_{n}^{e}(s ; c) \equiv \frac{1}{\Gamma(s)} \int_{0}^{\infty} d t t^{s-1} \exp \left(-t c^{2}\right) I_{n}^{e}(t),
$$

with $\zeta_{n, \alpha}^{r}$ defined exactl as above, with $I_{n}^{e}$ replaced with $I_{n, \alpha}$. There is no change in the contribution of $\zeta^{e}$ to the determinant from the untwisted case so we shall not analyze it any further. An analysis similar to that in [1] yields:

$$
\begin{aligned}
\zeta_{n, \alpha}^{r}(s ; c)= & \int_{0}^{\infty} \frac{d \lambda[\lambda(\lambda+2 c)]^{-s}}{\Gamma(s) \Gamma(1-s)} \sum_{\gamma \text { prim. } p=0} \sum_{p=0}^{\infty} \frac{l_{\gamma} v_{\gamma}^{2 n p} \operatorname{tr} \alpha\left(\gamma^{p}\right) \exp \left(p l_{\gamma}(-\lambda-c+1 / 2)\right)}{\exp \left(p l_{\gamma}\right)-1} \\
& \cdot 2\left[\cosh \left(n-\frac{1}{2}\right) p l_{\gamma}-v_{\gamma}^{p} \cosh n p l_{\gamma}\right] .
\end{aligned}
$$

Define a twisted Selberg zeta function for $\alpha$ as

$$
Z_{k, \alpha}(s) \equiv \operatorname{det} \prod_{\gamma \text { prim. }} \prod_{p=0}^{\infty}\left[1-v_{\gamma}^{2 k} \alpha(\gamma) \exp \left(-(s+p) l_{\gamma}\right)\right] \text { for } k \in \mathbb{Z} / 2 .
$$

We assume that $\eta$ is positive definite, so $S O(V, \eta)$ is compact, hence the trace is bounded. The above expression for $Z_{k, \alpha}$ then makes sense for $\Re s>1$. Observe that

$$
\frac{d}{d s} \ln Z_{k, \alpha}(s)=\sum_{\gamma \text { prim. }} \sum_{p=1}^{\infty} \frac{l_{\gamma} v_{\gamma}^{2 k p} \operatorname{tr} \alpha\left(\gamma^{p}\right) \exp \left((1-s) p l_{\gamma}\right)}{\exp \left(p l_{\gamma}\right)-1},
$$

so that we may write

$$
\begin{aligned}
\zeta_{n, \alpha}^{r}(s ; c)= & \int_{0}^{\infty} \frac{d \lambda[\lambda(\lambda+2 c)]^{-s}}{\Gamma(s) \Gamma(1-s)} \\
& \cdot \frac{d}{d \lambda} \ln \left[\frac{Z_{n, \alpha}(n+\lambda+c) Z_{n, \alpha}(-n+\lambda+c+1)}{Z_{n-1 / 2, \alpha}(n+\lambda+c+1 / 2) Z_{n-1 / 2, \alpha}(-n+\lambda+c+1 / 2)}\right] .
\end{aligned}
$$
obtain

Putting this together with the contribution from the identity element, we finally

$$
\begin{aligned}
s \operatorname{det}\left(\Delta_{n, \alpha}+c^{2}\right)= & \exp \left(-\frac{d}{d s} \zeta_{n, \alpha}(s=0 ; c)\right)=\exp \left(\frac{d_{\alpha} \chi(s H / s \Gamma)}{2} \sum_{k=0}^{2|n|} \ln \left(c^{2}-k^{2}\right)\right) \\
& \frac{Z_{n, \alpha}(n+c) Z_{n, \alpha}(-n+c+1)}{Z_{n-1 / 2, \alpha}(n+c+1 / 2) Z_{n-1 / 2, \alpha}(-n+c+1 / 2)} .
\end{aligned}
$$

From this expression one may obtain $s \operatorname{det} \Delta_{n, \alpha}$ by analytic continuation in $c$, though it is necessary to remove any zeroes that may occur. The results for bosonic determinants obtained in $[2,6]$ can be extended to general tensors by a similar analysis. As in the supersymmetric case, the identity contribution is unchanged and the untwisted Selberg zeta function $Z_{n}(s)^{d_{\alpha}}$ is replaced by the twisted zeta function $Z_{n, \alpha}(s)$ in the formulae for $\operatorname{det} \Delta_{n, \alpha}$.

A comment on the vanishing of the determinant: when $\alpha$ is an irreducible, unitary and non-trivial representation of $\Gamma$, it is known that $\lambda=0$ is not an eigenvalue of the Laplacian, $\Delta_{0, \alpha}\left[4\right.$, p. 353]. Therefore, the order of vanishing of $Z_{0, \alpha}$ is equal to the multiplicity of the trivial representation in the representation, $\alpha$, of $s \Gamma$ on $V$, in other words, the dimension of the $\alpha(s \Gamma)$-invariant subspace of $V$. 
The Selberg zeta function satisfies a functional relation,

$$
Z(s)=x(s) Z(1-s), \quad \text { where } \quad x(s) \equiv \exp \left(-2 \pi \chi(H / \Gamma) \int_{0}^{s-1 / 2} d v v \tan \pi v\right) .
$$

This extends easily to the twisted bosonic zeta function, with $x$ replaced by $x^{d_{\alpha}}$, because $x$ originates in the contribution of the identity to the trace formula. We expect this to hold in the supersymmetric case as well, which would imply that $s \operatorname{det} \Delta_{n, \alpha}$ is roughly the square of a ratio of bosonic determinants [1].

We shall now consider the factorization properties of the Selberg zeta function, $Z_{n, \alpha}$, for twisted internal channels. This involves calculating the asymptotics of the zeta function as the length of a primitive geodesic tends to zero. In the following, $\alpha \equiv \alpha(\gamma) v_{\gamma}^{2 n}$. The relevant part of the Selberg zeta function is then

$$
\mathscr{Z} \equiv \operatorname{det} \prod_{k=0}^{\infty}[1-\alpha \exp (-(s+k) l)]\left[1-\alpha^{-1} \exp (-(s+k) l)\right],
$$

where $l$ is the length of the geodesic that is shrinking and $\alpha$ is the twist associated to going around this geodesic. Suppose that $\alpha$ is an element of finite order, so that we have $\alpha^{q}=1$, for some $0<q \in \mathbf{Z}$.

We shall calculate the leading asymptotics of $\mathscr{Z}$ as $l \downarrow 0$. For this purpose, taking logarithms on both sides, and resumming $[9,7]$, we find $-\ln \mathscr{Z} \sim S_{q} / l$, where

$$
\begin{aligned}
S_{q} & \equiv \sum_{n=1}^{\infty} \frac{\operatorname{tr}\left(\alpha^{n}+\alpha^{-n}\right)}{n^{2}} \equiv \frac{1}{q^{2}}\left[\sum_{p=1}^{q-1} \operatorname{tr} \alpha^{p} \sum_{m=-\infty}^{\infty} \frac{1}{(m+p / q)^{2}}+d_{\alpha} \sum_{m=-\infty, m \neq 0}^{\infty} \frac{1}{m^{2}}\right] \\
& =\frac{\pi^{2}}{q^{2}}\left[\sum_{p=1}^{q-1} \operatorname{tr} \alpha^{p} \csc ^{2} \frac{\pi p}{q}+\frac{d_{\alpha}}{3}\right] .
\end{aligned}
$$

With a little more work, we can derive the subleading corrections to obtain

$$
Z_{n, \alpha} \propto \mathscr{Z} \propto l^{-\delta_{\alpha}(2 s-1)} \exp \left(-S_{q} / l\right) \quad \text { as } \quad l \downarrow 0, \quad \text { for } \quad \mathfrak{R} s>1,
$$

where $\delta_{\alpha}$ is the dimension of the $\alpha$-invariant subspace of $V$. In what follows, we shall only use the leading contribution.

We restrict our analysis to bosonic orbifolds. The lowest dimension field that passes through the $\alpha$-twisted channel is the $\alpha$-twist field. Identify the pinching coordinate $|t| \sim \exp \left(-2 \pi^{2} / l\right)$. To obtain the dimension, $h_{\alpha}$, of the $\alpha$-twist field, we compare the degeneration behavior of the twisted and untwisted zeta functions:

$$
\left(\tilde{Z}_{1, \alpha}(1) Z_{1}^{\prime}(1)^{-d_{\alpha}}\right)^{-1 / 2} \sim|t|^{2 h_{\alpha}}
$$

where $\tilde{Z}$ is the twisted Selberg zeta function with the zero modes removed. We can then use (1) to derive

$$
h_{\alpha}=\frac{1}{8 q^{2}}\left[\sum_{p=1}^{q-1} \operatorname{tr}\left(1-\alpha^{p}\right) \csc ^{2} \frac{\pi p}{q}\right] .
$$

As an example, consider the $Z_{q}$ orbifold [8], where $\operatorname{tr} \alpha=2 \cos 2 \pi k / q$, and hence

$$
h_{\alpha}=\frac{1}{2 q^{2}} \sum_{p=1}^{q-1} \frac{\sin ^{2} \pi k p / q}{\sin ^{2} \pi p / q}=\frac{1}{2} \frac{k}{q}\left(1-\frac{k}{q}\right) \text {. }
$$


Of course, (2) applies to arbitrary groups. A pleasing feature of our calculation is the unified manner in which we are able to treat arbitrary twists, including non-Abelian twists. We believe that this is the first determination of the entire spectrum of dimensions of bosonic twist fields for a non-Abelian group.

In summary, we have calculated the twisted superdeterminants that are necessary to study the physics of arbitrary left-right symmetric orbifolds of Euclidean space, as far as the point group twists are concerned. We have found simple expressions for the dimensions of the twist fields that correspond to such orbifolds by studying the degeneration behavior of the determinants at the boundary of moduli space. Our work applies to arbitrary genus hyperbolic surfaces; these include all the surfaces that are needed to study the physics of string theory about perturbatively stable vacua.

Acknowledgements. K. A. would like to thank Eric D'Hoker for encouraging him to write this manuscript. K. A.'s work was supported by NSF grants PHY80-19754 and PHY86-13201. V. P.'s work was supported by NSF Grant PHY82-17853, supplemented by funds from NASA. V. P. would like to thank the Aspen Center for Physics for its hospitality.

\section{References}

1. Aoki, K.: Heat kernels and superdeterminants of Laplace operators on super Riemann surfaces. Commun. Math. Phys. 117, 405-429 (1988)

2. D’Hoker, E., Phong, D. H.: The geometry of string perturbation theory. Rev. Mod. Phys. 60,917-1065 (1988)

3. Baranov, A. M., Manin, Yu. I., Frolov, I. V., Schwarz, A. S.: A superanalog of the Selberg trace formula and multiloop contributions for fermionic strings. Commun. Math. Phys. 111, 373-392 (1987); The multiloop contribution in the fermionic string. Yad. Fiz. 43, 1053-1056 (1986)

4. Hejhal, D.: The Selberg trace formula for $\operatorname{PSL}(2, \mathbf{R})$. Lecture Notes in Mathematics, vols 548, 1001, Berlin, Heidelberg, New York: Springer 1976, 1983

5. Fischer, J.: An approach to the Selberg trace formula via the Selberg zeta function. Lecture Notes in Mathematics, vol. 1253. Berlin, Heidelberg, New York: Springer 1987

6. Goncharov, Yu. P., Spokoiny, B.: Determinants of Laplacians in real line bundles connected with quantum geometry of strings. Lett. Math. Phys. 16, 263-268 (1988); Periwal, V.: Character twists. Nucl. Phys. B303, 94-98 (1988)

7. Wolpert, S. A.: Asymptotics of the spectrum and the Selberg zeta function on the space of Riemann surfaces. Commun. Math. Phys. 112, 283-315 (1987)

8. Hamidi, S., Vafa, C.: Interactions on orbifolds. Nucl. Phys. B279, 465-513 (1987); Dixon, L. J., Friedan, D. H., Martinec, E. J., Shenker, S. H.: The conformal field theory of orbifolds. Nucl. Phys. B282, 13-73 (1987)

9. Gava, E., Iengo, R., Jayaraman, T., Ramachandran, R.: Multiloop divergences in the closed bosonic string theory. Phys. Lett. 168B, 207-211 (1986)

Communicated by S.-T. Yau

Received December 9, 1988 